\title{
Boundary value problems for fractional $q$-difference equations with nonlocal conditions
}

\author{
Xinhui Li, Zhenlai Han*, Shurong Sun and Hongling Lu
}

\section{"Correspondence:}

hanzhenlai@163.com

School of Mathematical Sciences,

University of Jinan, Jinan, Shandong

250022, P.R. China

\begin{abstract}
In this paper, we study the boundary value problem of a fractional $q$-difference equation with nonlocal conditions involving the fractional $q$-derivative of the Caputo type, and the nonlinear term contains a fractional q-derivative of Caputo type. By means of Bananch's contraction mapping principle and Schaefer's fixed-point theorem, some existence results for the solutions are obtained. Finally, examples are presented to illustrate our main results.
\end{abstract}

MSC: 39A13; 34B18; 34A08

Keywords: fractional $q$-difference equations; boundary value problems; existence of solutions

\section{Introduction}

The $q$-difference calculus is an interesting and old subject. The $q$-difference calculus or quantum calculus was first developed by Jackson $[1,2]$, while basic definitions and properties can be found in the papers $[3,4]$. The origin of the fractional $q$-difference calculus can be traced back to the work in $[5,6]$ by Al-Salam and by Agarwal. The $q$-difference calculus describes many phenomena in various fields of science and engineering [1].

The $q$-difference calculus is an important part of discrete mathematics. More recently, the fractional $q$-difference calculus has caused wide attention of scholars, many researchers devoted themselves to studying it [7-14]. The relevant theory of fractional $q$-difference calculus has been established [15], such as $q$-analogues of the fractional integral and the difference operators properties as Mitlagel Leffler function [16], $q$-Laplace transform, $q$-Taylor's formula $[17,18]$, just to mention some. It is not only the requirements of the fractional $q$-difference calculus theory but also its the broad application.

Apart from this old history of $q$-difference equations, the subject received a considerable interest of many mathematicians and from many aspects, theoretical and practical. So specifically, $q$-difference equations have been widely used in mathematical physical problems, for dynamical system and quantum models [19], for $q$-analogues of mathematical physical problems including heat and wave equations [20], for sampling theory of signal analysis [21,22]. What is more, the fractional $q$-difference calculus plays an important role in quantum calculus.

As generalizations of integer order $q$-difference, fractional $q$-difference can describe physical phenomena much better and more accurately. Perhaps due to the development of

\section{黛 Springer}

○2014 Li et al.; licensee Springer. This is an Open Access article distributed under the terms of the Creative Commons Attribution License (http://creativecommons.org/licenses/by/2.0), which permits unrestricted use, distribution, and reproduction in any medium, provided the original work is properly cited. 
fractional differential equations [23-25], an interest has been aroused in studying boundary value problems of fractional $q$-difference equations, especially as regards the existence of solutions for boundary value problems [3, 4, 26-33].

In 2010, Ferreira [3] considered the existence of nontrivial solutions to the fractional $q$-difference equation

$$
\left(D_{q}^{\alpha} y\right)(x)=-f(x, y(x)), \quad 0<x<1,
$$

subject to the boundary conditions

$$
y(0)=0, \quad y(1)=0,
$$

where $1<\alpha \leq 2$ and $f:[0,1] \times \mathbb{R} \rightarrow \mathbb{R}$ is a nonnegative continuous function.

In 2011, El-Shahed and Al-Askar [27] studied the existence of a positive solution for the boundary value problem of the nonlinear factional $q$-difference equation

$$
{ }_{c} D_{q}^{\alpha} u+a(t) f(t)=0, \quad 0 \leq t \leq 1,2<\alpha \leq 3,
$$

with the boundary conditions

$$
u(0)=D_{q}^{2} u(0)=0, \quad \gamma D_{q} u(1)+\beta D_{q}^{2} u(1)=0,
$$

where $\gamma, \beta \leq 0$ and ${ }_{c} D_{q}^{\alpha}$ is a fractional $q$-derivative of Caputo type.

In 2012, Liang and Zhang [26] studied the existence and uniqueness of positive solutions for the three-point boundary problem of fractional $q$-differences

$$
\begin{aligned}
& \left(D_{q}^{\alpha} u\right)(t)+f(t, u(t))=0, \quad 0<t<1,2<\alpha<3, \\
& u(0)=\left(D_{q} u\right)(0)=0, \quad\left(D_{q} u\right)(1)=\beta\left(D_{q} u\right)(\eta),
\end{aligned}
$$

where $0<\beta \eta^{\alpha-2}<1$. By using a fixed-point theorem in partially ordered sets, they got some sufficient conditions for the existence and uniqueness of positive solutions to the above boundary problem.

In 2013, Zhou and Liu [28] considered the existence of solutions for the boundary value problems of the following nonlinear fractional $q$-difference equations:

$$
\begin{aligned}
& { }_{c} D_{q}^{\alpha} u+f(t, u)=0, \quad t \in J=[0,1], 2<\alpha \leq 3, \\
& u(0)=\left(D_{q}^{2} u\right)(0)=0, \quad \gamma\left(D_{q} u\right)(1)+\beta D_{q}^{2} u(1)=0,
\end{aligned}
$$

where $\gamma, \beta \geq 0$ and ${ }_{c} D_{q}$ is the fractional $q$-derivative of Caputo type. By virtue of Mönch's fixed-point theorem and the technique of measure of weak noncompactness and got some conditions of positive solutions.

In 2013, Zhou et al. [32] studied the existence results for fractional $q$-difference equations with nonlocal $q$-integral boundary conditions,

$$
\begin{aligned}
& \left(D_{q}^{\alpha} u\right)(t)+f(t, u(t))=0, \quad t \in(0,1), \\
& u(0)=0, \quad u(1)=\mu I_{q}^{\beta} u(\eta)=\mu \int_{0}^{\eta} \frac{(\eta-q s)^{(\beta-1)}}{\Gamma_{q}(\beta)} u(s) d_{q} s,
\end{aligned}
$$


where $\mu>0$ is a parameter, $D_{q}^{\alpha}$ is the $q$-derivative of Rieman-Liouville type of order $\alpha$. By using the generalized Banach contraction principle, the monotone iterative method and Krasnoselskii's fixed-point theorem, some existence results of positive solutions to the above boundary value problems have been enunciated.

In 2013, Li et al. [33] investigated the existence of solutions for the following two-point boundary value problem of nonlinear fractional $q$-difference equations:

$$
\begin{aligned}
& \left(D_{q}^{\alpha} u\right)(x)+\lambda f(u(x))=0, \quad 0<x<1, \\
& u(0)=D_{q} u(0)=D_{q} u(1)=0,
\end{aligned}
$$

where $0<q<1,2<\alpha<3, f: C((0,1),(0, \infty))$. They proved the existence of positive solutions for boundary value problems by utilizing a fixed-point theorem in cones. Several existence results for positive solutions in terms of different values of the parameter $\lambda$ were obtained.

In 2009, Benchohra et al. [34] studied the boundary value problem for fractional differential equations with nonlocal conditions

$$
\begin{aligned}
& { }^{c} D_{0+}^{\alpha} y(t)=f(t, y(t)), \quad t \in J=[0, T], 1<\alpha<2, \\
& y(0)=g(y), \quad y(T)=y_{T},
\end{aligned}
$$

where ${ }^{c} D_{0_{+}}^{\alpha}$ is the Caputo fractional derivative, $f:[1, T] \times \mathbb{R} \rightarrow \mathbb{R}$ is a continuous function, $g: C(J, \mathbb{R}) \rightarrow \mathbb{R}$ is a continuous function, and $y_{T} \in \mathbb{R}$.

To the best of our knowledge, there are a few papers that consider the boundary value of nonlinear fractional $q$-difference equations with nonlocal conditions and in which the nonlinear term contains a fractional $q$-derivative of Caputo type. Theory and applications seem to be just being initiated. In this paper we investigate the existence of solutions for the following boundary value problem of nonlinear fractional $q$-difference equations:

$$
\left({ }^{c} D_{q}^{\alpha} x\right)(t)+f\left(t,{ }^{c} D_{q}^{\sigma} x(t)\right)=0, \quad 0<t<1,
$$

subject to the boundary conditions

$$
x(0)=y(x), \quad \gamma\left(D_{q} x\right)(1)-\beta D_{q}^{2} x(1)=0,
$$

where $0<q<1,1<\alpha<2,0<\sigma<1, \beta \gamma \geq 0$ and $\frac{(1-t)^{(\alpha-2)}}{(1-t)^{(\alpha-3)}} \geq \frac{[\alpha]_{q} \beta}{\gamma}, f: C((0,1) \times \mathbb{R})$, and $y$ is a continuous functional. The condition of $\gamma\left(D_{q} x\right)(1)-\beta D_{q}^{2} x(1)=0$ is representative and general; the conditions of $D_{q} x(1)=0$ in [29] and $D_{q} x(1)=\beta$ in [4] can be viewed as two special cases. We will prove the existence of solutions for the boundary value problem (1.1)-(1.2) by utilizing Banach's contraction mapping principle and Schaefer's fixed-point theorem. Several existence results for the solutions are obtained. This work is motivated by papers $[28,34]$.

The paper is organized as follows. In Section 2, we introduce some definitions of $q$-fractional integral and differential operator together with some basic properties and lemmas to prove our main results. In Section 3, we investigate the existence of solutions for the boundary value problem (1.1)-(1.2) by the Banach contraction mapping principle 
and Schaefer's fixed-point theorem. Moreover, some examples are given to illustrate our main results.

\section{Preliminaries}

In the following section, we collect some definitions and lemmas about the fractional $q$ integral and fractional $q$-derivative for the full theory for which one is referred to $[3,33]$.

Let $q \in(0,1)$ and define

$$
[a]_{q}=\frac{1-q^{a}}{1-q}, \quad a \in \mathbb{R}
$$

and

$$
(a ; q)_{\infty}=\prod_{i=0}^{\infty}\left(1-a q^{i}\right), \quad(a ; q)_{\alpha}=\frac{(a ; q)_{\infty}}{\left(a q^{\alpha} ; q\right)_{\infty}} \quad(a, \alpha \in \mathbb{R}) .
$$

The $q$-analogue of the power function $(a-b)^{n}$ with $n \in \mathbb{N}_{0}$ is

$$
(a-b)^{0}=1, \quad(a-b)^{n}=\prod_{k=0}^{n-1}\left(a-b q^{k}\right), \quad n \in \mathbb{N}, a, b \in \mathbb{R} .
$$

More generally, if $\alpha \in \mathbb{R}$, then

$$
(a-b)^{(\alpha)}=a^{\alpha} \prod_{n=0}^{\infty} \frac{a-b q^{n}}{a-b q^{\alpha+n}} .
$$

It is easy to see that $[a(t-s)]^{(\alpha)}=a^{\alpha}(t-s)^{(\alpha)}$. And note that, if $b=0$, then $a^{(\alpha)}=a^{\alpha}$.

The $q$-gamma function is defined by

$$
\Gamma_{q}(x)=\frac{(1-q)^{(x-1)}}{(1-q)^{x-1}}, \quad x \in \mathbb{R} \backslash\{0,-1,-2, \ldots\},
$$

and it satisfies $\Gamma_{q}(x+1)=[x]_{q} \Gamma_{q}(x)$.

The $q$-derivative of a function $f$ is here defined by

$$
\left(D_{q} f\right)(x)=\frac{f(x)-f(q x)}{(1-q) x}, \quad\left(D_{q} f\right)(0)=\lim _{x \rightarrow 0}\left(D_{q} f\right)(x), \quad \text { for } x \neq 0,
$$

and $q$-derivatives of higher order are defined by

$$
\left(D_{q}^{0} f\right)(x)=f(x) \quad \text { and } \quad\left(D_{q}^{n} f\right)(x)=D_{q}\left(D_{q}^{n-1} f\right)(x), \quad n \in \mathbb{N} .
$$

The $q$-integral of a function $f$ defined on the interval $[0, b]$ is given by

$$
\left(I_{q} f\right)(x)=\int_{0}^{x} f(t) d_{q} t=x(1-q) \sum_{n=0}^{\infty} f\left(x q^{n}\right) q^{n}, \quad x \in[0, b]
$$

If $a \in[0, b]$ and $f$ is defined on the interval $[0, b]$, its $q$-integral from $a$ to $b$ is defined by

$$
\int_{a}^{b} f(t) d_{q} t=\int_{0}^{b} f(t) d_{q} t-\int_{0}^{a} f(t) d_{q} t
$$


Similarly as done for derivatives, an operator $I_{q}^{n}$ can be defined,

$$
\left(I_{q}^{0} f\right)(x)=f(x) \quad \text { and } \quad\left(I_{q}^{n} f\right)(x)=I_{q}\left(I_{q}^{n-1} f\right)(x), \quad n \in \mathbb{N} .
$$

From the definition of $q$-integral and the properties of series, we can get the following results concerning $q$-integral, which are helpful in the proofs of our main results.

\section{Lemma 2.1}

(1) Iff and $g$ are $q$-integral on the interval $[a, b], \alpha \in \mathbb{R}, c \in[a, b]$, then

(i) $\int_{a}^{b}(f(t)+g(t)) d_{q} t=\int_{a}^{b} f(t) d_{q} t+\int_{a}^{b} g(t) d_{q} t$;

(ii) $\int_{a}^{b} \alpha f(t) d_{q} t=\alpha \int_{b}^{a} f(t) d_{q} t$;

(iii) $\int_{a}^{b} f(t) d_{q} t=\int_{a}^{c} f(t) d_{q} t+\int_{c}^{b} f(t) d_{q} t$.

(2) If $|f|$ is q-integral on the interval $[0, x]$, then $\left|\int_{0}^{x} f(t) d_{q} t\right| \leq \int_{0}^{x}|f(t)| d_{q} t$.

(3) Iff and $g$ are $q$-integral on the interval $[0, x], f(t) \leq g(t)$ for all $t \in[0, x]$, then $\int_{0}^{x} f(t) d_{q} t \leq \int_{0}^{x} g(t) d_{q} t$.

The fundamental theorem of calculus applies to the operators $I_{q}$ and $D_{q}$, i.e.,

$$
\left(D_{q} I_{q} f\right)(x)=f(x)
$$

and if $f$ is continuous at $x=0$, then

$$
\left(I_{q} D_{q} f\right)(x)=f(x)-f(0) .
$$

The basic properties of the $q$-integral operator and the $q$-differential operator can be found in the book [15].

We now point out three formulas that will be used later $\left({ }_{i} D_{q}\right.$ denotes the derivative with respect to variable $i$ )

$$
\begin{aligned}
& { }_{t} D_{q}(t-s)^{(\alpha)}=[\alpha]_{q}(t-s)^{(\alpha-1)}, \\
& \left({ }_{x} D_{q} \int_{0}^{x} f(x, t) d_{q} t\right)(x)=\int_{0}^{x}{ }_{x} D_{q} f(x, t) d_{q} t+f(q x, x) .
\end{aligned}
$$

Remark 2.1 We note that if $\alpha>0$ and $a \leq b \leq t$, then $(t-a)^{(\alpha)} \geq(t-b)^{(\alpha)}$.

Definition 2.1 [6] Let $\alpha \geq 0$ and $f$ be a function defined on $[0, b]$. The fractional $q$-integral of the Riemann-Liouville type is $\left(I_{q}^{0} f\right)(x)=f(x)$ and

$$
\left(I_{q}^{\alpha} f\right)(x)=\frac{1}{\Gamma_{q}(\alpha)} \int_{0}^{x}(x-q t)^{(\alpha-1)} f(t) d_{q} t, \quad \alpha>0, x \in[0, b] .
$$

Definition 2.2 [16] The fractional $q$-derivative of the Caputo type of order $\alpha>0$ is defined by

$$
\left({ }^{c} D_{q}^{\alpha} f\right)(x)=\left(I_{q}^{[\alpha]-\alpha} D_{q}^{\lceil\alpha\rceil} f\right)(x), \quad \alpha>0,
$$

where $\lceil\alpha\rceil$ is the smallest integer greater than or equal to $\alpha$. 
Next, we list some properties of the $q$-derivative and the $q$-integral that are already known in the literature.

Lemma 2.2 [6] Let $\alpha, \beta \geq 0$ and $f$ be a function defined on $[0, b]$. Then the following formulas hold:

(i) $\left(I_{q}^{\beta} I_{q}^{\alpha} f\right)(x)=\left(I_{q}^{\alpha+\beta} f\right)(x)$;

(ii) $\left(D_{q}^{\alpha} I_{q}^{\alpha} f\right)(x)=f(x)$.

Lemma 2.3 [16] Let $\alpha \in \mathbb{R}^{+} \backslash \mathbb{N}$ and $a<x$, then the following is valid:

$$
\left(I_{q}^{\alpha c} D_{q}^{\alpha} f\right)(x)=f(x)-\sum_{k=0}^{\lceil\alpha\rceil-1} \frac{\left(D_{q}^{k} f\right)(a)}{\Gamma_{q}(k+1)} x^{k}(a / x ; q)_{k} .
$$

Lemma 2.4 [35] Let $B$ be a Banach space with $C \subseteq B$ closed and convex. Assume $U$ is a relatively open subset of $C$ with $0 \in U$ and $F: \bar{U} \rightarrow C$ is a continuous, compact map. Then either

(1) F has a fixed point in $\bar{U}$; or

(2) there exist $u \in \partial U$ and $\lambda \in(0,1)$ with $u=\lambda F u$.

The next result is important in the sequel.

Lemma 2.5 Let $1<\alpha<2$ and $h$ is continuous functional, a function $x$ is a solution of the fractional integral equation

$$
\begin{aligned}
x(t)= & y(x)+\left(\int_{0}^{1}\left(\frac{(1-q s)^{(\alpha-2)}}{\Gamma_{q}(\alpha-1)}-\frac{\beta(1-q s)^{(\alpha-3)}}{\gamma \Gamma_{q}(\alpha-2)}\right) h(s) d_{q} s\right) t \\
& -\int_{0}^{t} \frac{(t-q s)^{(\alpha-1)}}{\Gamma_{q}(\alpha)} h(s) d_{q} s,
\end{aligned}
$$

if and only if $x$ is a solution of the equation

$$
\begin{aligned}
& \left({ }^{c} D_{q}^{\alpha} x\right)(t)+h(t)=0, \quad 0<t<1, \\
& x(0)=y(x), \quad \gamma\left(D_{q} x\right)(1)-\beta D_{q}^{2} x(1)=0 .
\end{aligned}
$$

Proof By Definition 2.2 and Lemma 2.3, we can reduce (2.2) to an equivalent integral equation

$$
x(t)=x(0)+A t-I_{q}^{\alpha} h(t)
$$

where $A=\frac{D_{q} x(0)}{\Gamma_{q}(2)}$. Applying the boundary conditions $x(0)=y(x)$, we have

$$
x(t)=y(x)+A t-\int_{0}^{t} \frac{(t-q s)^{(\alpha-1)}}{\Gamma_{q}(\alpha)} h(s) d_{q} s .
$$

Thus, we obtain

$$
\left(D_{q} x\right)(t)=A-\int_{0}^{t} \frac{[\alpha-1]_{q}(t-q s)^{(\alpha-2)}}{\Gamma_{q}(\alpha)} h(s) d_{q} s,
$$




$$
\left(D_{q}^{2} x\right)(t)=-\int_{0}^{t} \frac{[\alpha-1]_{q}[\alpha-2]_{q}(t-q s)^{(\alpha-3)}}{\Gamma_{q}(\alpha)} h(s) d_{q} s .
$$

Next by the condition $\gamma\left(D_{q} x\right)(1)-\beta D_{q}^{2} x(1)=0$, we have

$$
A=\int_{0}^{1} \frac{(1-q s)^{(\alpha-2)}}{\Gamma_{q}(\alpha-1)} h(s) d_{q} s-\int_{0}^{1} \frac{\beta(1-q s)^{(\alpha-3)}}{\gamma \Gamma_{q}(\alpha-2)} h(s) d_{q} s .
$$

Therefore, the unique solution of the problem (2.2)-(2.3) is

$$
x(t)=y(x)+\left(\int_{0}^{1}\left(\frac{(1-q s)^{(\alpha-2)}}{\Gamma_{q}(\alpha-1)}-\frac{\beta(1-q s)^{(\alpha-3)}}{\gamma \Gamma_{q}(\alpha-2)}\right) h(s) d_{q} s\right) t-\int_{0}^{t} \frac{(t-q s)^{(\alpha-1)}}{\Gamma_{q}(\alpha)} h(s) d_{q} s,
$$

which completes the proof.

\section{Main results}

We are now in a position to state and prove our main results in this paper.

Let $B=\left\{x \mid x \in C[0,1]\right.$ and $\left.D_{q} x \in C[0,1]\right\}$ be the Banach space endowed with the norm $\|x\|=\max _{t \in[0,1]}\left\{|x(t)|,\left|{ }^{c} D_{q}^{\sigma} x(t)\right|\right\}$. Define the closed subset $K \subset B$ by $K=\{x(t) \in C[0,1] \mid$ $x(t) \geq 0\}$.

Define the operator $F: K \rightarrow K$ by

$$
\begin{aligned}
(F x)(t)= & y(x)+\left(\int_{0}^{1}\left(\frac{(1-q s)^{(\alpha-2)}}{\Gamma_{q}(\alpha-1)}-\frac{\beta(1-q s)^{(\alpha-3)}}{\gamma \Gamma_{q}(\alpha-2)}\right) f\left(s,{ }^{c} D_{q}^{\sigma} x(s)\right) d_{q} s\right) t \\
& -\int_{0}^{t} \frac{(t-q s)^{(\alpha-1)}}{\Gamma_{q}(\alpha)} f\left(s,{ }^{c} D_{q}^{\sigma} x(s)\right) d_{q} s, \quad t \in[0,1] .
\end{aligned}
$$

Obviously, the fixed points of the operator $F$ are solutions of the boundary value problem (1.1)-(1.2).

For convenience, we define

$$
\begin{aligned}
& C_{1}=\int_{0}^{1}\left(\frac{(1-q s)^{(\alpha-2)}}{\Gamma_{q}(\alpha-1)}-\frac{\beta(1-q s)^{(\alpha-3)}}{\gamma \Gamma_{q}(\alpha-2)}\right) d_{q} s, \\
& C_{2}=\int_{0}^{1} \frac{(1-q s)^{(\alpha-1)}}{\Gamma_{q}(\alpha)} d_{q} s .
\end{aligned}
$$

Then we have the following results.

\section{Theorem 3.1 Assume that}

(H1) there exists a constant $l_{1}>0$ such that $\left|f\left(t, z_{2}\right)-f\left(t, z_{1}\right)\right| \leq l_{1}\left(\left|z_{2}-z_{1}\right|\right)$, for each $t \in[0,1]$ and all $z_{1}, z_{2} \in \mathbb{R}$;

(H2) there exists a constant $l_{2}>0$ such that $\left|y\left(x_{2}\right)-y\left(x_{1}\right)\right| \leq l_{2}\left\|x_{2}-x_{1}\right\|$, for each $x_{1}, x_{2} \in K$

(H3) $\theta=\max \left\{l_{2}+l_{1}\left(C_{1}+C_{2}\right), l_{1}\left(C_{1}+\frac{[\alpha-1]_{q}}{\Gamma_{q}(\alpha)} C_{3}\right) \frac{C_{4}}{\Gamma_{q}(1-\sigma)}\right\}<1$, where $C_{3}$ and $C_{4}$ are defined as (3.8) and (3.9).

Then the boundary value problem (1.1)-(1.2) has at least one positive solution. 
Proof Let $x_{1}, x_{2} \in K$, then for all $t \in[0,1]$, the following inequality holds:

$$
\begin{aligned}
& \left|\left(F x_{2}\right)(t)-\left(F x_{1}\right)(t)\right| \\
& \leq\left|\int_{0}^{1}\left(\frac{(1-q s)^{(\alpha-2)}}{\Gamma_{q}(\alpha-1)}-\frac{\beta(1-q s)^{(\alpha-3)}}{\gamma \Gamma_{q}(\alpha-2)}\right)\left(f\left(s,{ }^{c} D_{q}^{\sigma} x_{2}(s)\right)-f\left(s,{ }^{c} D_{q}^{\sigma} x_{1}(s)\right)\right) d_{q} s\right| \\
& \quad+\left|\int_{0}^{t} \frac{(t-q s)^{(\alpha-1)}}{\Gamma_{q}(\alpha)}\left(f\left(s,{ }^{c} D_{q}^{\sigma} x_{2}(s)\right)-f\left(s,{ }^{c} D_{q}^{\sigma} x_{1}(s)\right)\right) d_{q} s\right|+\left|y\left(x_{2}\right)-y\left(x_{1}\right)\right| \\
& \leq \int_{0}^{1}\left(\frac{(1-q s)^{(\alpha-2)}}{\Gamma_{q}(\alpha-1)}-\frac{\beta(1-q s)^{(\alpha-3)}}{\gamma \Gamma_{q}(\alpha-2)}\right)\left|f\left(s,{ }^{c} D_{q}^{\sigma} x_{2}(s)\right)-f\left(s,{ }^{c} D_{q}^{\sigma} x_{1}(s)\right)\right| d_{q} s \\
& \quad+\int_{0}^{t} \frac{(t-q s)^{(\alpha-1)}}{\Gamma_{q}(\alpha)}\left|f\left(s,{ }^{c} D_{q}^{\sigma} x_{2}(s)\right)-f\left(s,{ }^{c} D_{q}^{\sigma} x_{1}(s)\right)\right| d_{q} s+\left|y\left(x_{2}\right)-y\left(x_{1}\right)\right| .
\end{aligned}
$$

By (H1) and (H2), we may arrange (3.2) as follows:

$$
\begin{aligned}
\left|\left(F x_{2}\right)(t)-\left(F x_{1}\right)(t)\right| & \\
\leq & l_{2}\left\|x_{2}-x_{1}\right\|+\int_{0}^{1}\left(\frac{(1-q s)^{(\alpha-2)}}{\Gamma_{q}(\alpha-1)}-\frac{\beta(1-q s)^{(\alpha-3)}}{\gamma \Gamma_{q}(\alpha-2)}\right) l_{1}\left|{ }^{c} D_{q}^{\sigma} x_{2}(s)-{ }^{c} D_{q}^{\sigma} x_{1}(s)\right| d_{q} s \\
& \quad+\int_{0}^{t} \frac{(t-q s)^{(\alpha-1)}}{\Gamma_{q}(\alpha)} l_{1}\left|{ }^{c} D_{q}^{\sigma} x_{2}(s)-{ }^{c} D_{q}^{\sigma} x_{1}(s)\right| d_{q} s \\
\leq & l_{2}\left\|x_{2}-x_{1}\right\|+\int_{0}^{1}\left(\frac{(1-q s)^{(\alpha-2)}}{\Gamma_{q}(\alpha-1)}-\frac{\beta(1-q s)^{(\alpha-3)}}{\gamma \Gamma_{q}(\alpha-2)}\right) l_{1}\left\|{ }^{c} D_{q}^{\sigma} x_{2}-{ }^{c} D_{q}^{\sigma} x_{1}\right\| d_{q} s \\
& +\int_{0}^{1} \frac{(1-q s)^{(\alpha-1)}}{\Gamma_{q}(\alpha)} l_{1}\left\|{ }^{c} D_{q}^{\sigma} x_{2}-{ }^{c} D_{q}^{\sigma} x_{1}\right\| d_{q} s \\
\leq & l_{2}\left\|x_{2}-x_{1}\right\|+\left(C_{1}+C_{2}\right) l_{1}\left\|{ }^{c} D_{q}^{\sigma} x_{2}-{ }^{c} D_{q}^{\sigma} x_{1}\right\| \\
\leq & l_{2}\left\|x_{2}-x_{1}\right\|+\left(C_{1}+C_{2}\right) l_{1}\left\|x_{2}-x_{1}\right\| \\
= & \left(l_{2}+l_{1}\left(C_{1}+C_{2}\right)\right)\left\|x_{2}-x_{1}\right\| .
\end{aligned}
$$

As

$$
\begin{aligned}
D_{q}(F x)(t)= & \int_{0}^{1} \frac{(1-q s)^{(\alpha-2)}}{\Gamma_{q}(\alpha-1)}-\frac{\beta(1-q s)^{(\alpha-3)}}{\gamma \Gamma_{q}(\alpha-2)} f\left(s,{ }^{c} D_{q}^{\sigma} x(s)\right) d_{q} s \\
& -\int_{0}^{t} \frac{[\alpha-1]_{q}(t-q s)^{(\alpha-2)}}{\Gamma_{q}(\alpha)} f\left(s,{ }^{c} D_{q}^{\sigma} x(s)\right) d_{q} s,
\end{aligned}
$$

by the Definition 2.2 , here $0<\sigma<1,\lceil\sigma\rceil=1$, and

$$
\begin{aligned}
\left({ }^{c} D_{q}^{\sigma} F x\right)(t) & =\left(I_{q}^{\lceil\sigma\rceil-\sigma} D_{q}^{\lceil\sigma\rceil} F x\right)(t) \\
& =\int_{0}^{t} \frac{(t-q s)^{(\lceil\sigma\rceil-\sigma-1)}}{\Gamma_{q}(\lceil\sigma\rceil-\sigma)}\left(D_{q}^{\lceil\sigma\rceil} F x\right)(s) d_{q} s \\
& =\int_{0}^{t} \frac{(t-q s)^{(-\sigma)}}{\Gamma_{q}(1-\sigma)}\left(D_{q} F x\right)(s) d_{q} s .
\end{aligned}
$$


We can get the following deduction:

$$
\begin{aligned}
& \left|D_{q}\left(F x_{2}\right)(t)-D_{q}\left(F x_{1}\right)(t)\right| \\
& \leq\left|\int_{0}^{1}\left(\frac{(1-q s)^{(\alpha-2)}}{\Gamma_{q}(\alpha-1)}-\frac{\beta(1-q s)^{(\alpha-3)}}{\gamma \Gamma_{q}(\alpha-2)}\right)\left(f\left(s,{ }^{c} D_{q}^{\sigma} x_{2}(s)\right)-f\left(s,{ }^{c} D_{q}^{\sigma} x_{1}(s)\right)\right) d_{q} s\right| \\
& \quad+\left|\int_{0}^{t} \frac{[\alpha-1]_{q}(t-q s)^{(\alpha-2)}}{\Gamma_{q}(\alpha)} f\left(s,{ }^{c} D_{q}^{\sigma} x_{2}(s)\right)-f\left(s,{ }^{c} D_{q}^{\sigma} x_{1}(s)\right) d_{q} s\right| \\
& \leq \int_{0}^{1}\left(\frac{(1-q s)^{(\alpha-2)}}{\Gamma_{q}(\alpha-1)}-\frac{\beta(1-q s)^{(\alpha-3)}}{\gamma \Gamma_{q}(\alpha-2)}\right)\left|f\left(s,{ }^{c} D_{q}^{\sigma} x_{2}(s)\right)-f\left(s,{ }^{c} D_{q}^{\sigma} x_{1}(s)\right)\right| d_{q} s \\
& \quad+\int_{0}^{t} \frac{[\alpha-1]_{q}(t-q s)^{(\alpha-2)}}{\Gamma_{q}(\alpha)}\left|f\left(s,{ }^{c} D_{q}^{\sigma} x_{2}(s)\right)-f\left(s,{ }^{c} D_{q}^{\sigma} x_{1}(s)\right)\right| d_{q} s .
\end{aligned}
$$

By (H1) and (H2), we arrange (3.5) as follows:

$$
\begin{aligned}
& \left|D_{q}\left(F x_{2}\right)(t)-D_{q}\left(F x_{1}\right)(t)\right| \\
& \leq \int_{0}^{1}\left(\frac{(1-q s)^{(\alpha-2)}}{\Gamma_{q}(\alpha-1)}-\frac{\beta(1-q s)^{(\alpha-3)}}{\gamma \Gamma_{q}(\alpha-2)}\right) l_{1}\left\|{ }^{c} D_{q}^{\sigma} x_{2}-{ }^{c} D_{q}^{\sigma} x_{1}\right\| d_{q} s \\
& \quad+\int_{0}^{t} \frac{[\alpha-1]_{q}(t-q s)^{(\alpha-2)}}{\Gamma_{q}(\alpha)} l_{1}\left\|{ }^{c} D_{q}^{\sigma} x_{2}-{ }^{c} D_{q}^{\sigma} x_{1}\right\| d_{q} s \\
& \leq \int_{0}^{1}\left(\frac{(1-q s)^{(\alpha-2)}}{\Gamma_{q}(\alpha-1)}-\frac{\beta(1-q s)^{(\alpha-3)}}{\gamma \Gamma_{q}(\alpha-2)}\right) l_{1}\left\|{ }^{c} D_{q}^{\sigma} x_{2}-{ }^{c} D_{q}^{\sigma} x_{1}\right\| d_{q} s \\
& \quad+\frac{[\alpha-1]_{q}(1-q)}{\Gamma_{q}(\alpha)} t^{(\alpha-1)} \sum_{n=0}^{\infty}\left(1-q^{n+1}\right)^{(\alpha-2)} q^{n} l_{1}\left\|{ }^{c} D_{q}^{\sigma} x_{2}-{ }^{c} D_{q}^{\sigma} x_{1}\right\| d_{q} s \\
& \leq\left(C_{1} l_{1}+\frac{[\alpha-1]_{q}(1-q)}{\Gamma_{q}(\alpha)} \sum_{n=0}^{\infty}\left(1-q^{n+1}\right)^{(\alpha-2)} q^{n} l_{1}\right)\left\|{ }^{c} D_{q}^{\sigma} x_{2}-{ }^{c} D_{q}^{\sigma} x_{1}\right\| d_{q} s .
\end{aligned}
$$

By the theory of series, let

$$
\begin{aligned}
a_{n} & =\left(1-q^{n+1}\right)^{(\alpha-2)} q^{n}=\prod_{k=0}^{\infty} \frac{1-q^{n+k+1}}{1-q^{n+k+\alpha-1}} q^{n} \\
& =q^{n} e^{\sum_{k=0}^{\infty} \ln \left(1-q^{n+k+1}\right)-\ln \left(1-q^{n+k+\alpha-1}\right)}, \\
b_{n} & =\sum_{k=0}^{\infty}\left(\ln \left(1-q^{n+k+1}\right)-\ln \left(1-q^{n+k+\alpha-1}\right)\right) .
\end{aligned}
$$

Note that $0<q<1$, so $\sum_{k=0}^{\infty} q^{n+k+1}$ and $\sum_{k=0}^{\infty} q^{n+k+\alpha+1}$ are convergent, which imply that $\sum_{k=0}^{\infty} \ln \left(1-q^{n+k+1}\right)$ and $\sum_{k=0}^{\infty} \ln \left(1-q^{n+k+\alpha-1}\right)$ are convergent. Thus $\sum_{k=0}^{\infty} b_{n}$ is convergent. Hence $\sum_{n=0}^{\infty} a_{n}=\sum_{n=0}^{\infty}\left(1-q^{n+1}\right)^{(\alpha-2)} q^{n}$ is convergent.

Setting

$$
C_{3}=\sum_{n=0}^{\infty}\left(1-q^{n+1}\right)^{(\alpha-2)} q^{n}
$$


and combining with (3.6), we get

$$
\left|D_{q}\left(F x_{2}\right)(t)-D_{q}\left(F x_{1}\right)(t)\right| \leq\left(C_{1}+\frac{[\alpha-1]_{q}(1-q)}{\Gamma_{q}(\alpha)} C_{3}\right) l_{1}\left\|{ }^{c} D_{q}^{\sigma} x_{2}-{ }^{c} D_{q}^{\sigma} x_{1}\right\| .
$$

So,

$$
\begin{aligned}
& \left|\left({ }^{c} D_{q}^{\sigma} F x_{2}\right)(t)-\left({ }^{c} D_{q}^{\sigma} F x_{1}\right)(t)\right| \\
& \leq \int_{0}^{t} \frac{(t-q s)^{(-\sigma)}}{\Gamma_{q}(1-\sigma)}\left(C_{1}+\frac{[\alpha-1]_{q}(1-q)}{\Gamma_{q}(\alpha)} C_{3}\right) l_{1}\left\|{ }^{c} D_{q}^{\sigma} x_{2}-{ }^{c} D_{q}^{\sigma} x_{1}\right\| d_{q} s \\
& =l_{1}\left(C_{1}+\frac{[\alpha-1]_{q}(1-q)}{\Gamma_{q}(\alpha)} C_{3}\right)\left\|{ }^{c} D_{q}^{\sigma} x_{2}-{ }^{c} D_{q}^{\sigma} x_{1}\right\| \frac{t(1-q) \sum_{n=0}^{\infty}\left(t-q^{n+1} t\right)^{(-\sigma)} q^{n}}{\Gamma_{q}(1-\sigma)} \\
& =l_{1}\left(C_{1}+\frac{[\alpha-1]_{q}(1-q)}{\Gamma_{q}(\alpha)} C_{3}\right)\left\|{ }^{c} D_{q}^{\sigma} x_{2}-{ }^{c} D_{q}^{\sigma} x_{1}\right\| \\
& \quad \times \frac{t^{(1-\sigma)}(1-q) \sum_{n=0}^{\infty}\left(1-q^{n+1}\right)^{(-\sigma)} q^{n}}{\Gamma_{q}(1-\sigma)} .
\end{aligned}
$$

Choosing $u_{n}=\left(1-q^{n+1}\right)^{(-\sigma)} q^{n}$, we see that $\sum_{n=0}^{\infty} u_{n}=\sum_{n=0}^{\infty}\left(1-q^{n+1}\right)^{(-\sigma)} q^{n}$ is convergent, and we may as well set

$$
C_{4}=\sum_{n=0}^{\infty}\left(1-q^{n+1}\right)^{(-\sigma)} q^{n}
$$

and combining with (3.8), we get

$$
\begin{aligned}
\left|\left({ }^{c} D_{q}^{\sigma} F x_{2}\right)(t)-\left({ }^{c} D_{q}^{\sigma} F x_{1}\right)(t)\right| & \leq l_{1}\left(C_{1}+\frac{[\alpha-1]_{q}(1-q)}{\Gamma_{q}(\alpha)} C_{3}\right) \frac{(1-q)}{\Gamma_{q}(1-\sigma)} C_{4}\left\|x_{2}-x_{1}\right\| \\
& \leq l_{1}\left(C_{1}+\frac{[\alpha-1]_{q}}{\Gamma_{q}(\alpha)} C_{3}\right) \frac{C_{4}}{\Gamma_{q}(1-\sigma)}\left\|x_{2}-x_{1}\right\| .
\end{aligned}
$$

It follows that

$$
\begin{aligned}
\left\|F x_{2}-F x_{1}\right\| & =\max _{t \in[0,1]}\left\{\left|F\left(x_{2}\right)(t)-F\left(x_{1}\right)(t)\right|,\left|{ }^{c} D_{q}^{\sigma} F\left(x_{2}\right)(t)-{ }^{c} D_{q}^{\sigma} F\left(x_{1}\right)(t)\right|\right\} \\
& \leq \theta\left\|x_{2}-x_{1}\right\| .
\end{aligned}
$$

Consequently $F$ is a contraction map as $\theta<1$. As a consequence of Banach's fixed-point theorem, we deduce that $F$ has a fixed point which is a solution of the problem (1.1)-(1.2). The proof is completed.

Denote

$$
\begin{aligned}
& M_{1}=\max _{t \in[0,1]}|y(x(t))|, \quad M_{2}=\max _{t \in[0,1]}\left|f\left(t,{ }^{c} D_{q}^{\sigma} x(t)\right)\right|, \\
& A=M_{1}+M_{2}\left(C_{1}+C_{2}\right), \quad B=\frac{C_{4} M_{2}}{\Gamma_{q}(1-\sigma)}\left(C_{1}+\frac{[\alpha-1]_{q} C_{3}}{\Gamma_{q}(\alpha)}\right) .
\end{aligned}
$$


Theorem 3.2 Assume $D_{q} x(t)$ and $f:[0,1] \times \mathbb{R} \rightarrow \mathbb{R}$ are continuous, and $y>0$ is a continuous functional. Suppose the following conditions are satisfied:

(H4) there exists a continuous function $\varphi:(0,+\infty) \rightarrow \mathbb{R}$ with $|f(t, z)| \leq \varphi(z)$ on $[0,1] \times(0,+\infty)$

(H5) there exists $r>0$, with $\|\varphi\| \leq \frac{r-M_{1}}{C_{1}+C_{2}}$.

Then the boundary value problem (1.1)-(1.2) has a solution.

Proof We will prove the result by using Schaefer's fixed-point theorem and divide the proof into four steps.

First, set $U=\{x \mid x \in K,\|x\|<r\}$, then $\bar{U}=\{x \mid x \in K,\|x\| \leq r\}$, we show $F: \bar{U} \rightarrow K$ is continuous.

Since $D_{q} x(t)$ is continuous, then ${ }^{c} D_{q}^{\sigma} x(t)=\int_{0}^{t} \frac{(1-q s)(-\sigma)}{\Gamma_{q}(1-\sigma)} D_{q} x(s) d_{q} s$ is continuous. Choosing $\left\{x_{n}\right\}$ to be a sequence such that $x_{n} \rightarrow x$ in $\bar{U}$, then

$$
\begin{aligned}
\left|\left(F x_{n}\right)(t)-(F x)(t)\right| & \\
\leq & \int_{0}^{1}\left(\frac{(1-q s)^{(\alpha-2)}}{\Gamma_{q}(\alpha-1)}-\frac{\beta(1-q s)^{(\alpha-3)}}{\gamma \Gamma_{q}(\alpha-2)}\right)\left|f\left(s,{ }^{c} D_{q}^{\sigma} x_{n}(s)\right)-f\left(s,{ }^{c} D_{q}^{\sigma} x(s)\right)\right| d_{q} s \\
& +\int_{0}^{t} \frac{(t-q s)^{(\alpha-1)}}{\Gamma_{q}(\alpha)}\left|f\left(s,{ }^{c} D_{q}^{\sigma} x_{n}(s)\right)-f\left(s,{ }^{c} D_{q}^{\sigma} x(s)\right)\right| d_{q} s+\left|y\left(x_{n}\right)-y(x)\right| \\
\leq & \int_{0}^{1}\left(\frac{(1-q s)^{(\alpha-2)}}{\Gamma_{q}(\alpha-1)}-\frac{\beta(1-q s)^{(\alpha-3)}}{\gamma \Gamma_{q}(\alpha-2)}\right) \max _{s \in[0,1]}\left|f\left(s,{ }^{c} D_{q}^{\sigma} x_{n}(s)\right)-f\left(s,{ }^{c} D_{q}^{\sigma} x(s)\right)\right| d_{q} s \\
& +\int_{0}^{t} \frac{(t-q s)^{(\alpha-1)}}{\Gamma_{q}(\alpha)} \max _{s \in[0,1]}\left|f\left(s,{ }^{c} D_{q}^{\sigma} x_{n}(s)\right)-f\left(s,{ }^{c} D_{q}^{\sigma} x(s)\right)\right| d_{q} s+\left|y\left(x_{n}\right)-y(x)\right| .
\end{aligned}
$$

From $f$ and $y$ are continuous, we have

$$
\left\|F\left(x_{n}\right)-F(x)\right\| \rightarrow 0 \quad \text { as } n \rightarrow \infty .
$$

Now, we prove that $F: \bar{U} \rightarrow K$ is a compact map. For any $0<\eta \leq r$, set $E=\{x \in K \mid\|x\| \leq$ $\eta\}$, it suffices to show that $F(E)$ is relatively compact set in $K$. In fact, for each $t \in[0,1]$,

$$
\begin{aligned}
|(F x)(t)|= & \mid y(x)+\left(\int_{0}^{1}\left(\frac{(1-q s)^{(\alpha-2)}}{\Gamma_{q}(\alpha-1)}-\frac{\beta(1-q s)^{(\alpha-3)}}{\gamma \Gamma_{q}(\alpha-2)}\right) f\left(s,{ }^{c} D_{q}^{\sigma} x(s)\right) d_{q} s\right) t \\
& -\int_{0}^{t} \frac{(t-q s)^{(\alpha-1)}}{\Gamma_{q}(\alpha)} f\left(s,{ }^{c} D_{q}^{\sigma} x(s)\right) d_{q} s \mid \\
\leq & |y(x)|+\int_{0}^{1}\left(\frac{(1-q s)^{(\alpha-2)}}{\Gamma_{q}(\alpha-1)}-\frac{\beta(1-q s)^{(\alpha-3)}}{\gamma \Gamma_{q}(\alpha-2)}\right)\left|f\left(s,{ }^{c} D_{q}^{\sigma} x(s)\right)\right| d_{q} s \\
& +\int_{0}^{t} \frac{(t-q s)^{(\alpha-1)}}{\Gamma_{q}(\alpha)}\left|f\left(s,{ }^{c} D_{q}^{\sigma} x(s)\right)\right| d_{q} s \\
\leq & M_{1}+M_{2} \int_{0}^{1} \frac{(1-q s)^{(\alpha-2)}}{\Gamma_{q}(\alpha-1)}-\frac{\beta(1-q s)^{(\alpha-3)}}{\gamma \Gamma_{q}(\alpha-2)} d_{q} s+M_{2} \int_{0}^{1} \frac{(1-q s)^{(\alpha-1)}}{\Gamma_{q}(\alpha)} d_{q} s \\
\leq & M_{1}+M_{2}\left(C_{1}+C_{2}\right)=A .
\end{aligned}
$$


We consider

$$
\begin{aligned}
\left|D_{q}(F x)(t)\right|= & \mid \int_{0}^{1}\left(\frac{(1-q s)^{(\alpha-2)}}{\Gamma_{q}(\alpha-1)}-\frac{\beta(1-q s)^{(\alpha-3)}}{\gamma \Gamma_{q}(\alpha-2)}\right) f\left(s,{ }^{c} D_{q}^{\sigma} x(s)\right) d_{q} s \\
& -\int_{0}^{t} \frac{[\alpha-1]_{q}(t-q s)^{(\alpha-2)}}{\Gamma_{q}(\alpha)} f\left(s,{ }^{c} D_{q}^{\sigma} x(s)\right) d_{q} s \mid \\
\leq & \int_{0}^{1}\left(\frac{(1-q s)^{(\alpha-2)}}{\Gamma_{q}(\alpha-1)}-\frac{\beta(1-q s)^{(\alpha-3)}}{\gamma \Gamma_{q}(\alpha-2)}\right)\left|f\left(s,{ }^{c} D_{q}^{\sigma} x(s)\right)\right| d_{q} s \\
& +\int_{0}^{t} \frac{[\alpha-1]_{q}(t-q s)^{(\alpha-2)}}{\Gamma_{q}(\alpha)}\left|f\left(s,{ }^{c} D_{q}^{\sigma} x(s)\right)\right| d_{q} s \\
= & {\left[\int_{0}^{1}\left(\frac{(1-q s)^{(\alpha-2)}}{\Gamma_{q}(\alpha-1)}-\frac{\beta(1-q s)^{(\alpha-3)}}{\gamma \Gamma_{q}(\alpha-2)}\right) d_{q} s\right.} \\
& \left.+\frac{[\alpha-1]_{q}(1-q)}{\Gamma_{q}(\alpha)} t^{(\alpha-1)} \sum_{n=0}^{\infty}\left(1-q^{n+1}\right)^{(\alpha-2)} q^{n}\right]\left|f\left(s,{ }^{c} D_{q}^{\sigma} x(s)\right)\right| \\
\leq & M_{2}\left(C_{1}+\frac{[\alpha-1]_{q}}{\Gamma_{q}(\alpha)} \sum_{n=0}^{\infty}\left(1-q^{n+1}\right)^{(\alpha-2)} q^{n}\right) \\
\leq & M_{2}\left(C_{1}+\frac{[\alpha-1]_{q} C_{3}}{\Gamma_{q}(\alpha)}\right) .
\end{aligned}
$$

We get

$$
\begin{aligned}
\left|\left({ }^{c} D_{q}^{\sigma} F x\right)(t)\right| & =\left|\int_{0}^{t} \frac{(t-q s)^{(-\sigma)}}{\Gamma_{q}(1-\beta)} D_{q} F(x) d_{q} s\right| \\
& \leq M_{2}\left(C_{1}+\frac{[\alpha-1]_{q} C_{3}}{\Gamma_{q}(\alpha)}\right) \int_{0}^{t} \frac{(t-q s)^{(-\sigma)}}{\Gamma_{q}(1-\sigma)} d_{q} s \\
& =M_{2}\left(C_{1}+\frac{[\alpha-1]_{q} C_{3}}{\Gamma_{q}(\alpha)}\right) t(1-q) \sum_{n=0}^{\infty} \frac{\left(t-t q^{n+1}\right)^{(-\sigma)}}{\Gamma_{q}(1-\sigma)} q^{n} \\
& \leq \frac{C_{4} M_{2}}{\Gamma_{q}(1-\sigma)}\left(C_{1}+\frac{[\alpha-1]_{q} C_{3}}{\Gamma_{q}(\alpha)}\right)=B .
\end{aligned}
$$

For each $t \in[0,1]$, we have

$$
\begin{aligned}
& \|F x\| \leq M_{1}+M_{2}\left(C_{1}+C_{2}\right)=A, \\
& \left\|\left({ }^{c} D_{q}^{\sigma} F x\right)\right\| \leq \frac{C_{4} M_{2}}{\Gamma_{q}(1-\sigma)}\left(C_{1}+\frac{[\alpha-1]_{q} C_{3}}{\Gamma_{q}(\alpha)}\right)=B .
\end{aligned}
$$

Hence, we conclude that

$$
\|F x\|=\max \left\{|(F x)(t)|,\left|{ }^{c} D_{q}^{\sigma}(F x)(t)\right|\right\} \leq l=\max \{A, B\},
$$

which shows $F(E)$ is uniform bounded.

On the other hand, for any given $\varepsilon>0$, setting

$$
\delta=\min \left\{\frac{1}{2}, \frac{\varepsilon}{M_{2}\left(C_{1}+2 C_{2}\right)}\right\},
$$


for each $x \in E, 0 \leq t_{1} \leq t_{2} \leq 1$ and $\left|t_{2}-t_{1}\right|<\delta$, one has $\left|(F x)\left(t_{2}\right)-(F x)\left(t_{1}\right)\right|<\varepsilon$, that is to say, $F(E)$ is equicontinuous. In fact,

$$
\begin{aligned}
& \left|(F x)\left(t_{2}\right)-(F x)\left(t_{1}\right)\right| \\
& \leq \int_{0}^{1}\left(\frac{(1-q s)^{(\alpha-2)}}{\Gamma_{q}(\alpha-1)}-\frac{\beta(1-q s)^{(\alpha-3)}}{\gamma \Gamma_{q}(\alpha-2)}\right)\left|f\left(s,{ }^{c} D_{q}^{\sigma} x(s)\right)\right| d_{q} s\left(t_{2}-t_{1}\right) \\
& \quad+\left|\int_{0}^{t_{2}} \frac{\left(t_{2}-q s\right)^{(\alpha-1)}}{\Gamma_{q}(\alpha)} f\left(s,{ }^{c} D_{q}^{\sigma} x(s)\right) d_{q} s-\int_{0}^{t_{1}} \frac{\left(t_{1}-q s\right)^{(\alpha-1)}}{\Gamma_{q}(\alpha)} f\left(s,{ }^{c} D_{q}^{\sigma} x(s)\right) d_{q} s\right| \\
& \leq \\
& \quad M_{2} \int_{0}^{1}\left(\frac{(1-q s)^{(\alpha-2)}}{\Gamma_{q}(\alpha-1)}-\frac{\beta(1-q s)^{(\alpha-3)}}{\gamma \Gamma_{q}(\alpha-2)}\right) d_{q} s\left(t_{2}-t_{1}\right) \\
& \quad+\frac{M_{2}}{\Gamma_{q}(\alpha)}\left|\int_{0}^{t_{2}}\left(t_{2}-q s\right)^{(\alpha-1)} d_{q} s-\int_{0}^{t_{1}}\left(t_{1}-q s\right)^{(\alpha-1)} d_{q} s\right| \\
& \leq M_{2} \int_{0}^{1}\left(\frac{(1-q s)^{(\alpha-2)}}{\Gamma_{q}(\alpha-1)}-\frac{\beta(1-q s)^{(\alpha-3)}}{\gamma \Gamma_{q}(\alpha-2)}\right) d_{q} s\left(t_{2}-t_{1}\right) \\
& \quad+\frac{M_{2}}{\Gamma_{q}(\alpha)}\left|t_{2}^{(\alpha)} \int_{0}^{1}(1-q s)^{(\alpha-1)} d_{q} s-t_{1}^{(\alpha)} \int_{0}^{1}(1-q s)^{(\alpha-1)} d_{q} s\right| \\
& =M_{2} C_{1}\left(t_{2}-t_{1}\right)+M_{2} C_{2}\left|t_{2}^{(\alpha)}-t_{1}^{(\alpha)}\right| .
\end{aligned}
$$

Now, we estimate $t_{2}^{(\alpha)}-t_{1}^{(\alpha)}$ :

(1) for $0 \leq t_{1}<\delta, \delta \leq t_{2}<2 \delta, t_{2}^{(\alpha)}-t_{1}^{(\alpha)} \leq t_{2}^{(\alpha)}<(2 \delta)^{(\alpha)} \leq 2 \delta$;

(2) for $0 \leq t_{1}<t_{2} \leq \delta, t_{2}^{(\alpha)}-t_{1}^{(\alpha)} \leq t_{2}^{(\alpha-1)}<\delta^{(\alpha-1)} \leq 2 \delta$;

(3) for $\delta \leq t_{1}<t_{2} \leq 1$. From the mean value theorem of differentiation, we have $t_{2}^{(\alpha)}-$ $t_{1}^{(\alpha)} \leq[\alpha]\left(t_{2}-t_{1}\right) \leq 2 \delta$. Thus, we have

$$
\left|F x\left(t_{2}\right)-F x\left(t_{1}\right)\right|<M_{2} C_{1} \delta+2 M_{2} C_{2} \delta<\varepsilon .
$$

Therefore, $F(E)$ is equicontinuous. By means of the Arzela-Ascoli theorem, $F(E)$ is a relatively compact set in $K$, then the operator $F: \bar{U} \rightarrow K$ is completely continuous.

In the fourth step, we have a priori bounds.

Suppose $x \in \partial U$ is a solution of

$$
x(t)=\lambda F x(t)
$$

for $\lambda \in(0,1)$, where $F$ is given by (3.1). We know that $F: \bar{U} \rightarrow K$ is continuous and completely continuous. Furthermore, by $(\mathrm{H} 4)$ and $(\mathrm{H} 5)$ we have

$$
\begin{aligned}
x(t)= & \lambda F x(t) \\
= & \lambda\left(y(x)+\left[\int_{0}^{1}\left(\frac{(1-q s)^{(\alpha-2)}}{\Gamma_{q}(\alpha-1)}-\frac{\beta(1-q s)^{(\alpha-3)}}{\gamma \Gamma_{q}(\alpha-2)}\right) f\left(s,{ }^{c} D_{q}^{\sigma} x(s)\right) d_{q} s\right] t\right. \\
& \left.-\int_{0}^{t} \frac{(t-q s)^{(\alpha-1)}}{\Gamma_{q}(\alpha)} f\left(s,{ }^{c} D_{q}^{\sigma} x(s)\right) d_{q} s\right) \\
< & y(x)+\int_{0}^{1}\left(\frac{(1-q s)^{(\alpha-2)}}{\Gamma_{q}(\alpha-1)}-\frac{\beta(1-q s)^{(\alpha-3)}}{\gamma \Gamma_{q}(\alpha-2)}\right) \varphi\left({ }^{c} D_{q}^{\sigma} x(s)\right) d_{q} s
\end{aligned}
$$




$$
\begin{aligned}
& \quad+\int_{0}^{t} \frac{(t-q s)^{(\alpha-1)}}{\Gamma_{q}(\alpha)} \varphi\left({ }^{c} D_{q}^{\sigma} x(s)\right) d_{q} s \\
& \leq y(x)+\int_{0}^{1}\left(\frac{(1-q s)^{(\alpha-2)}}{\Gamma_{q}(\alpha-1)}-\frac{\beta(1-q s)^{(\alpha-3)}}{\gamma \Gamma_{q}(\alpha-2)}\right) \varphi\left({ }^{c} D_{q}^{\sigma} x(s)\right) d_{q} s \\
& +\int_{0}^{1} \frac{(1-q s)^{(\alpha-1)}}{\Gamma_{q}(\alpha)} \varphi\left({ }^{c} D_{q}^{\sigma} x(s)\right) d_{q} s \\
& =M_{1}+\|\varphi\|\left(C_{1}+C_{2}\right) \leq r,
\end{aligned}
$$

where $r$ is defined as (H5). It follows that $\|x\|<r$, that is, there is no $x \in \partial U$ such that $x=\lambda F(x)$ for $\lambda \in(0,1)$. As a consequence of Lemma $2.4, F$ has a fixed point $x \in \bar{U}$ which is a solution of the boundary value problem (1.1)-(1.2), and the proof is completed.

\section{Examples}

In this section, we present some examples to illustrate our main results.

Example 4.1 Consider the following boundary value problem:

$$
\begin{aligned}
& { }^{c} D_{q}^{\frac{5}{4}} x(t)+f\left(t,{ }^{c} D_{q}^{\frac{1}{3}} x(t)\right)=0, \\
& x(0)=y(x)=c, \quad \gamma\left(D_{q} x\right)(1)-\beta D_{q}^{2} x(1)=0,
\end{aligned}
$$

here $\alpha=\frac{5}{4}, \sigma=\frac{1}{3}, \beta=0$, and $c$ is a constant. Note that

$$
\begin{aligned}
& C_{1}=\int_{0}^{1} \frac{(1-q s)^{\left(-\frac{3}{4}\right)}}{\Gamma_{q}\left(\frac{1}{4}\right)} d_{q} s, \quad C_{2}=\int_{0}^{1} \frac{(1-q s)^{\left(\frac{1}{4}\right)}}{\Gamma_{q}\left(\frac{5}{4}\right)} d_{q} s, \\
& C_{3}=\sum_{n=0}^{\infty}\left(1-q^{n+1}\right)^{\left(-\frac{3}{4}\right)} q^{n} d_{q} s, \quad C_{4}=\sum_{n=0}^{\infty}\left(1-q^{n+1}\right)^{\left(-\frac{1}{3}\right)} q^{n} d_{q} s .
\end{aligned}
$$

For convenience, we denote $A_{1}=\frac{C_{4}}{\Gamma_{q}(1-\sigma)}\left(C_{1}+\frac{[\alpha-1]_{q}}{\Gamma_{q}(\alpha)} C_{3}\right), A_{2}=\left(C_{1}+C_{2}\right)$. Choosing $A_{3}>$ $\max \left\{A_{1}, A_{2}\right\}$, let $l_{1}=\frac{1}{A_{3}}>0, l_{2}=1-\frac{A_{2}}{A_{3}}>0$, and we get $\theta=\max \left\{l_{1} A_{1}, l_{2}+l_{1}\left(C_{1}+C_{2}\right)\right\}<1$. When $f\left(t,{ }^{c} D_{q}^{\frac{1}{3}} x(t)\right)=l_{1}{ }^{c} D_{q}^{\frac{1}{3}} x(t)$ and $y(x)=c$, for any $t_{1}, t_{2} \in[0,1], x_{1}, x_{2} \in K$, the following equalities hold:

$$
\mid f\left(t_{1},{ }^{c} D_{q}^{\frac{1}{3}} x\left(t_{1}\right)-f\left(t_{2},{ }^{c} D_{q}^{\frac{1}{3}} x\left(t_{2}\right)\right) \mid=l_{1}\left(\left|{ }^{c} D_{q}^{\frac{1}{3}} x\left(t_{1}\right)-{ }^{c} D_{q}^{\frac{1}{3}} x\left(t_{2}\right)\right|\right),\right.
$$

and

$$
\left|y\left(x_{1}\right)-y\left(x_{2}\right)\right|=0 .
$$

Hence, by Theorem 3.1, the boundary value problem (4.1)-(4.2) has a solution.

Example 4.2 Consider the following boundary value problem:

$$
{ }^{c} D_{q}^{\frac{3}{2}} x(t)-\frac{\Gamma_{q}\left(\frac{3}{2}\right)\left(t \sin \left({ }^{c} D_{q}^{\frac{1}{2}} x(t)\right)\right)^{2}}{\int_{0}^{1}\left[\frac{1}{2}\right]_{q}(1-q s)^{\left(-\frac{1}{2}\right)} d_{q} s+\int_{0}^{1}(1-q s)^{\left(\frac{1}{2}\right)} d_{q} s}=0,
$$




$$
x(0)=y(x)=\sin x(t), \quad \gamma\left(D_{q} x\right)(1)-\beta D_{q}^{2} x(1)=0,
$$

here $\alpha=\frac{3}{2}, \beta=0, \sigma=\frac{1}{2}, f\left(t,{ }^{c} D^{\frac{1}{2}} x(t)\right)=-\frac{\Gamma_{q}\left(\frac{3}{2}\right)\left(t \sin \left({ }^{c} D_{q}^{\frac{1}{2}} x(t)\right)\right)^{2}}{\int_{0}^{1}\left[\frac{1}{2}\right]_{q}(1-q s)^{\left(-\frac{1}{2}\right)} d_{q} s+\int_{0}^{1}(1-q s)^{\left(\frac{1}{2}\right)} d_{q} s}, x(0)=y(x)=\sin x(t)$ and

$$
C_{1}=\int_{0}^{1} \frac{(1-q s)^{\left(-\frac{1}{2}\right)}}{\Gamma_{q}\left(\frac{1}{2}\right)} d_{q} s, \quad C_{2}=\int_{0}^{1} \frac{(1-q s)^{\left(\frac{1}{2}\right)}}{\Gamma_{q}\left(\frac{3}{2}\right)} d_{q} s
$$

Note that

$$
\begin{aligned}
& M_{1}=\max _{t \in[0,1]}|y(x(t))|=1, \\
& \varphi\left({ }^{c} D_{q}^{\frac{1}{2}} x(t)\right)=\frac{1}{C_{1}+C_{2}}=\frac{\Gamma_{q}\left(\frac{3}{2}\right)}{\int_{0}^{1}\left[\frac{1}{2}\right]_{q}(1-q s)^{\left(-\frac{1}{2}\right)} d_{q} s+\int_{0}^{1}(1-q s)^{\left(\frac{1}{2}\right)} d_{q} s},
\end{aligned}
$$

and we get $\left|f\left(t,{ }^{c} D^{\frac{1}{2}} x(t)\right)\right| \leq \varphi\left({ }^{c} D_{q}^{\frac{1}{2}} x(t)\right)$ with $\|\varphi\|=\frac{1}{C_{1}+C_{2}}$. Choosing $r=3$, it is clear that $\|\varphi\| \leq \frac{r-M}{C_{1}+C_{2}}=\frac{2}{C_{1}+C_{2}}$. By Theorem 3.2, the boundary value problem (4.3)-(4.4) has a solution.

\section{Competing interests}

The authors declare that they have no competing interests.

\section{Authors' contributions}

The authors declare that the study was realized in collaboration with the same responsibility. All authors read and approved the final manuscript.

\section{Acknowledgements}

The authors sincerely thank the reviewers for their valuable suggestions and useful comments that have led to the present improved version of the original manuscript. This research is supported by the Natural Science Foundation of China (61374074), the Natural Science Outstanding Youth Foundation of Shandong Province (JQ201119) and supported by the Shandong Provincial Natural Science Foundation (ZR2012AM009, ZR2013AL003). It is also supported by the Graduate Innovation Foundation of University of Jinan (YCX13013).

Received: 9 November 2013 Accepted: 16 January 2014 Published: 04 Feb 2014

\section{References}

1. Jackson, F: On q-functions and a certain difference operator. Trans. R. Soc. Edinb. 46, 253-281 (1908)

2. Jackson, F: On q-definite integrals. Q. J. Pure Appl. Math. 41, 193-203 (1910)

3. Ferreira, R: Nontrivial solutions for fractional q-difference boundary value problems. Electron. J. Qual. Theory Differ. Equ. 70, 1-10 (2010)

4. Ferreira, R: Positive solutions for a class of boundary value problems with fractional $q$-differences. Comput. Math. Appl. 61, 367-373 (2011)

5. Al-Salam, W: Some fractional $q$-integrals and q-derivatives. Proc. Edinb. Math. Soc. 15(2), 135-140 (1966/1967)

6. Agarwal, R: Certain fractional q-integrals and q-derivatives. Proc. Camb. Philos. Soc. 66, 365-370 (1969)

7. Ahmad, B, Nieto, J: Basic theory of nonlinear third-order q-difference equations and inclusions. Math. Model. Anal. 18(1), 122-135 (2013)

8. Dobrogowska, A: The q-deformation of the Morse potential. Appl. Math. Lett. 26(7), 769-773 (2013)

9. Priya, G, Prakash, P, Nieto, J, Kayar, Z: Higher-order numerical scheme for the fractional heat equation with Dirichlet and Neumann boundary conditions. Numer. Heat Transf., Part B, Fundam. 63(6), 540-559 (2013)

10. Ahmad, B, Nietoa, J: Boundary value problems for a class of sequential integro differential equations of fractional order. J. Funct. Spaces Appl. 2013, Article ID 149659 (2013)

11. Ahmad, B, Nieto, J, Alsaedi, A, El-Shahed, M: A study of nonlinear Langevin equation involving two fractional orders in different intervals. Nonlinear Anal., Real World Appl. 13(2), 599-606 (2012)

12. Ahmad, B, Nieto, J: On nonlocal boundary value problems of nonlinear q-difference equations. Adv. Differ. Equ. 2012, Article ID 81 (2012)

13. Cruz, A, Martins, N, Torres, D: Higher-order Hahn's quantum variational calculus. Nonlinear Anal. 75(3), 1147-1157 (2012)

14. Goodrich, C: On discrete sequential fractional boundary value problems. J. Math. Anal. Appl. 385(1), 111-124 (2012) 
15. Kac, V, Cheung, P: Quantum Calculus. Springer, New York (2002)

16. Rajković, P, Marinković, S, Stanković, M: On q-analogues of Caputo derivative and Mittag-Leffler function. Fract. Calc. Appl. Anal. 4(10), 359-373 (2007)

17. Rajković, P, Marinković, S, Stanković, M: Fractional integrals and derivatives in q-calculus. Appl. Anal. Discrete Math $1(1), 311-323(2007)$

18. Atici, F, Eloe, P: Fractional q-calculus on a time scale. J. Nonlinear Math. Phys. 14(3), 341-352 (2007)

19. Abdel-Gawad, H, Aldailami, A: On q-dynamic equations modelling and complexity. Appl. Math. Model. 34(3), 697-709 (2010)

20. Field, C, Joshi, N, Nijhoff, F: q-difference equations of KdV type and Chazy-type second-degree difference equations. J. Phys. A, Math. Theor. 41(33), 332005 (2008)

21. Abdi, W: Certain inversion and representation formulae for q-Laplace transforms. Math. Z. 83, 238-249 (1964)

22. Abreu, L: Sampling theory associated with q-difference equations of the Sturm-Liouville type. J. Phys. A 38(48), 10311-10319(2005)

23. Zhang, S: Existence of solution for a boundary value problem of fractional order. Acta Math. Sci. 26, 220-228 (2006)

24. Zhao, Y, Sun, S, Han, Z, Li, Q: The existence of multiple positive solutions for boundary value problems of nonlinear fractional differential equations. Commun. Nonlinear Sci. Numer. Simul. 16(4), 2086-2097 (2011)

25. Feng, W, Sun, S, Han, Z, Zhao, Y: Existence of solutions for a singular system of nonlinear fractional differential equations. Comput. Math. Appl. 62(3), 1370-1378 (2011)

26. Liang, S, Zhang, J: Existence and uniqueness of positive solutions for three-point boundary value problem with fractional q-differences. J. Appl. Math. Comput. 40, 277-288 (2012)

27. El-Shahed, M, Al-Askar, F: Positive solution for boundary value problem of nonlinear fractional $q$-difference equation. ISRN Math. Anal. 2011, Article ID 385459 (2011)

28. Zhou, W, Liu, H: Existence solutions for boundary value problem of nonlinear fractional $q$-difference equations. Adv. Differ. Equ. 2013, Article ID 113 (2013)

29. Yang, L, Chen, H, Luo, L, Luo, Z: Successive iteration and positive solutions for boundary value problem of nonlinear fractional q-difference equation. J. Appl. Math. Comput. 42, 89-102 (2013)

30. Alsaedi, A, Ahmad, B, Al-Hutami, H: A study of nonlinear fractional q-difference equations with nonlocal integral boundary conditions. Abstr. Appl. Anal. 2013, Article ID 928147 (2013)

31. Yu, C, Wang, J: Positive solutions of nonlocal boundary value problem for high-order nonlinear fractional $q$-difference equations. Abstr. Appl. Anal. 2013, Article ID 410505 (2013)

32. Zhao, Y, Chen, $\mathrm{H}$, Zhang, Q: Existence results for fractional $q$-difference equations with nonlocal $q$-integral boundary conditions. Adv. Differ. Equ. 2013, Article ID 48 (2013)

33. Li, X, Han, Z, Sun, S: Existence of positive solutions of nonlinear fractional q-difference equation with parameter. Adv. Differ. Equ. 2013, Article ID 260 (2013)

34. Benchohra, M, Hamaini, S, Notuyas, S: Boundary value problems for differential equations with fractional order and nonlocal conditions. Nonlinear Anal. 71, 2391-2396 (2009)

35. Granans, A, Guenther, P, Lee, J: Some general existence principle in Carathéodory theory of nonlinear systems. J. Math. Pures Appl. 70, 153-196 (1991)

10.1186/1687-1847-2014-57

Cite this article as: Li et al.: Boundary value problems for fractional $q$-difference equations with nonlocal conditions. Advances in Difference Equations 2014, 2014:57

\section{Submit your manuscript to a SpringerOpen ${ }^{\ominus}$ journal and benefit from:}

- Convenient online submission

Rigorous peer review

- Immediate publication on acceptance

- Open access: articles freely available online

- High visibility within the field

- Retaining the copyright to your article 